\section{New products and focus on digital dentistry and orthodontics}

\section{DIGITAL IMAGING TRAINING CENTRE OPENS}

Digital imaging company Vatech \&t E-Woo recently opened its new training facility in Feltham, Middlesex. A new initiative, the Imaging Centre's facilities are available to dental professionals and their teams. Its purpose is to help the profession better understand dental digital radiography, its potential and its capabilities.

The company, which manufactures products such as the PaX-Reve 3D, is also offering computed tomography user training courses for the dental

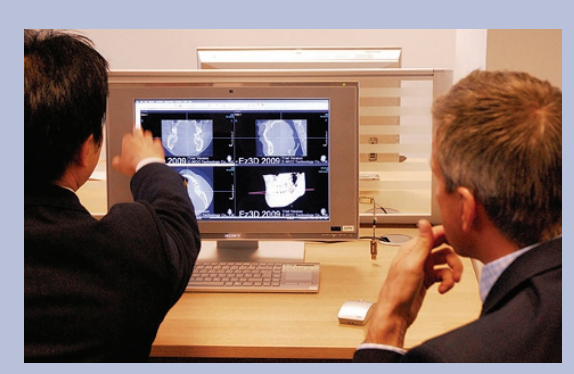

team. Upcoming course dates for 2009 are 26 June, 31 July, 28 August, 25 September, 30 October and 27 November. Reader response number 50

\title{
NEW TOOTHBRUSH FOR KIDS LAUNCHED
}

Philips has launched a new Sonicare toothbrush - Sonicare for Kids - aimed at children aged 4 to 10 years of age. The new brush is based on the core Sonicare technology but incorporates a number of new elements.

A KidTimer function helps paediatric patients reach the recommended two minute brushing time by progressively increasing the brushing duration over the course of 90 days.

The in-built timer and quadpacer has a musical tones indicator and because it is aimed at children, the new brush has two kid friendly power modes to ensure gentle yet effective cleaning.

At low mode it is $60 \%$ less powerful than Sonicare FlexCare making it ideal for children aged 4+ and at high mode it is $40 \%$ less powerful for children aged 7+ and has significantly less vibration and a lower noise level than its parent brush.

An ergonomic handle fits easily in the child's hand and includes two gripping locations for training purposes. Age

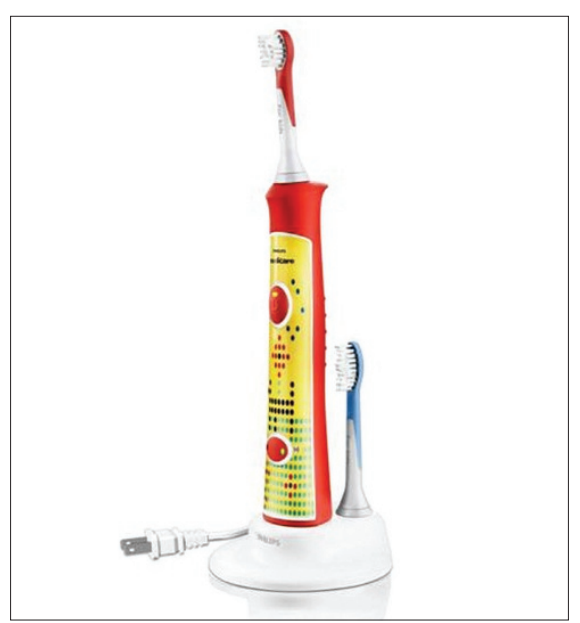

appropriate brush heads in two sizes also accommodate a child's development. These have a protective rubber overmould to shield tender young gums.

Sonicare for Kids also includes interchangeable decorative panels, an antiroll device, a large, easy to find on/off button and a sturdy charger base which incorporates a brush head holder.

Reader response number 51
Please send trade news information and illustrations to Arveen Bajaj at the $B D J$, Nature Publishing Group, The Macmillan Building, 4-6 Crinan Street, London N1 9XW. Trade news is provided as a service to readers using text and images from the manufacturer, supplier or distributor and does not imply endorsement by the $B D J$. Normal and prudent research should be exercised before purchase or use of any product mentioned.

\section{BACTERICIDAL BIODEGRADABLE} WIPES

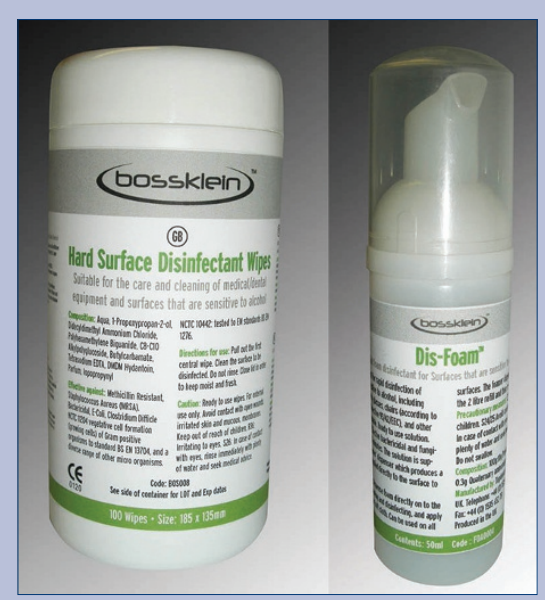

New alcohol-free Bossklein infection control products, available from Blackwell Supplies, have been designed specifically to achieve a high standard of cleanliness without causing the damage associated with many alcohol-based products.

Bossklein Wet Wipes have a 60-second bactericidal effect, and also combat adenovirus and enveloped viruses including hepatitis B, hepatitis C and HIV. Supplied fully sealed and completely fresh, these pleasantly scented and biodegradable wipes are ready to use straight from the pack.

Bossklein Foam takes effect against bacteria and fungi within ten minutes, and hepatitis $B$ and HIV within five minutes. Supplied in a unique, biodegradable $50 \mathrm{ml}$ dispenser, it offers easy application. The Foam is also effective against Clostridium difficile NCTC 11204 and the growing cells of grampositive organisms.

Reader response number 52 


\section{SCHOOLS TOOLKIT LAUNCHED}

The recently launched Listerine Clinical Educational Toolkit is an educational resource designed for use by dental hygiene and therapy tutors to support students throughout their years of study.

It comprises educational lectures, digital illustrations and graphics and plaque viability experimental materials and protocols. It contains the Listerine
Clinical Overview Booklet which is a collection of research abstracts that provide an at-a-glance reference to numerous clinical research papers.

The booklet also contains everyday practice scenarios suggesting different approaches to treatment according to the personal profile of the individual patient. It also concludes with a questionnaire providing one hour of CPD.

Reader response number 53

\section{INTEGRATED IMAGING}

Sirona offers an integrated concept consisting of 3D imaging as well as computer-aided implant planning and

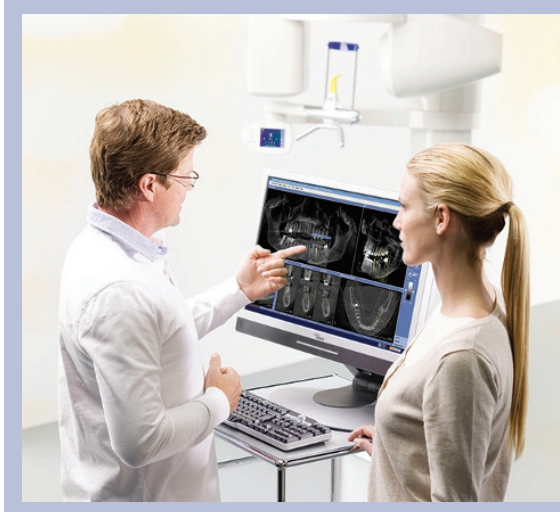

implementation. The Galileos system, in combination with Galaxis 3D X-ray software requires only one scan in order to visualise the entire cranial volume. The cone beam technology deployed in the system clearly reveals anatomical structures.

With the help of integrated software application Galileos Implant, the practitioner can plan the implants on the same user interface. This planning data, together with a plaster impression and bite registration, is then sent to the Sirona subsidiary siCAT in Bonn. In a matter of days the practitioner receives the custom-made surgical template. Reader response number 54

\section{DIGITAL IMPRESSIONS AT YOUR CHAIRSIDE}

With the Lava chairside intra-oral scanner (COS) recently launched in the UK, 3M ESPE hopes to fundamentally transform the interaction between dentists and technicians in the creation of indirect restorations, leading to more predictable and accurate results.

The Lava COS collects digital data directly from the patient's mouth by taking an impression using a wand measuring $13.2 \mathrm{~mm}$ wide at its tip - providing easy movement throughout the mouth.

An extra advantage of this technology is its ability to capture continuous three-dimensional video streams in the mouth and display the data on the touch screen in real time. This enables instant feedback for both the dentist and patient to see the anatomy of the mouth and specific areas of interest.

It is possible to view all parts of the impression precisely because the 3D images can be rotated and enlarged.
If required, additional data is captured and added to form increasingly detailed images. The completed digital model on the screen is used for interaction with the patient and as a basis for the production of an accurate restoration.

The set of captured digital data is electronically sent to the laboratory. Here, margins are marked and the die is virtually cut. The file is then transmitted to a model manufacturing facility that produces a precise stereolithography (SLA) model and to the laboratory for the production of either a Lava CAD/CAM or conventional (PFM) restoration.

In addition, 3M ESPE will also launch several new system components for laboratories and authorised Lava milling centres (ALMCs) in 2009. In combination with the Lava COS, these solutions will provide access to an entirely digital

\section{SENSITIVE TOOTH TREATMENT}

Savodent Sensitive available from Swallow Dental is a treatment for sensitive teeth which is quick and easy to use. Two active foam strips are placed in a double tray, which is then worn for ten minutes per treatment, giving immediate relief from the pain of sensitive teeth.

One single application gives relief for up to one month. The active ingredient, potassium oxalate, works to reduce pain by modifying the nerve excitability. Savodent provides significantly faster and longer lasting results than sensitive teeth toothpastes.

Reader response number 55

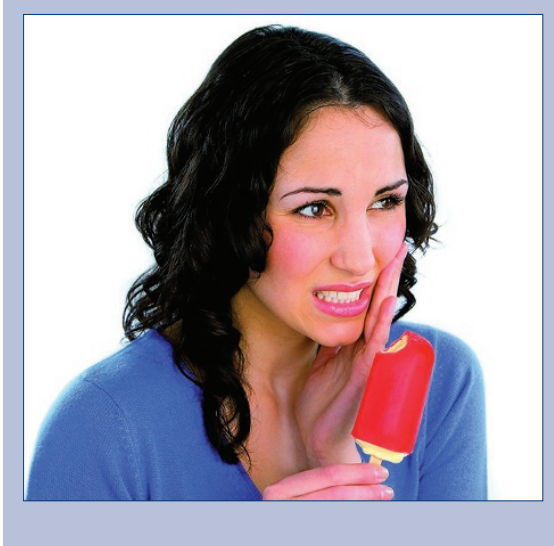

workflow for the production of indirect restorations, from impression taking to cementation.

Reader response number 56

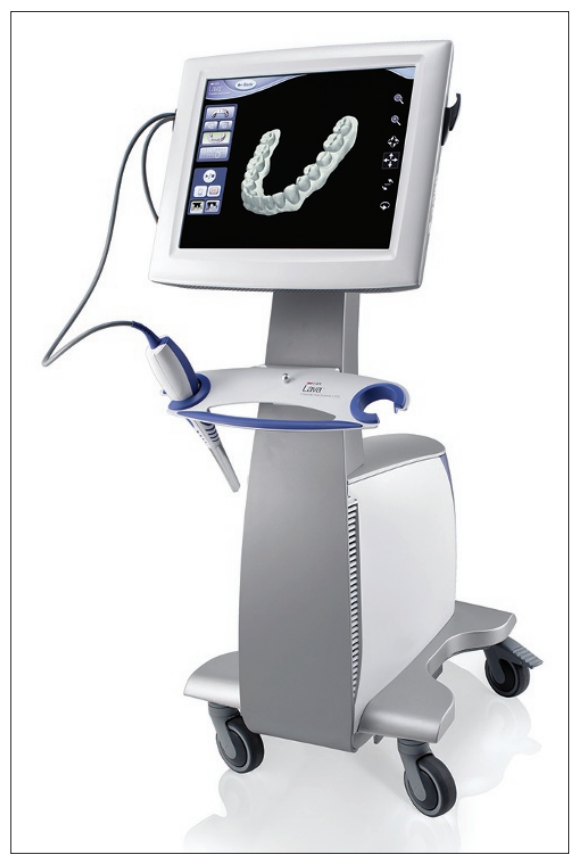




\section{PRECISELY PLAN IMPLANT TREATMENT}

The Gendex cone beam 3D dental imager GXCB-500 from KaVo is powered by i-CAT. It features an amorphous silicon flat panel sensor that delivers accurate images at a scan time of only 8.9 seconds with full 3D reconstruction in less than 20 seconds.

The single sensor design allows staff to switch from 3D to 2D imaging, saving time. It has a standard field of view of 8 $\times 8 \mathrm{~cm}$ while its special EDS shot enables a view of $14 \times 8 \mathrm{~cm}$

The equipment offers dental professionals a unique 'medium field of view' which enables immediate diagnostic information to allow dentists to precisely plan implant treatment.

The GXCB-500 can be used for implantology, with nerve canals easily identi-

\section{EFFICIENT DENTAL SYSTEM}

The new Duo dental system from Castellini has advanced features in ergonomics, accessibility and comfort and uses the latest in technology and design to create an efficient system to suit all dental practices.

The new Hanging Tube version features hanging tube instruments, a height-adjustable DSA table and a rotating, double-jointed operating table. Improved operating light features include an attenuation filter for photopolymerisation and an automatic shutoff function when the chair is reset.

The Duo caters for every working need and each unique feature is controlled from a practical and intuitive console. Operators have access to up to five fied by nerve marking estimation. The technology is easily incorporated into the practice and on-site training is available. Reader response number 57

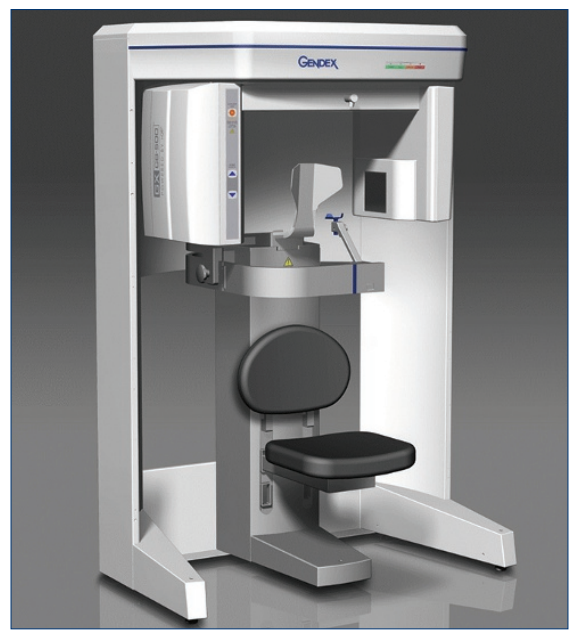

instruments, all on self-balancing levers to eliminate traction on the wrist.

When combined with the Dama chair, the Duo also provides both patient and operator with optimal positioning in all clinical situations.

Reader response number 58

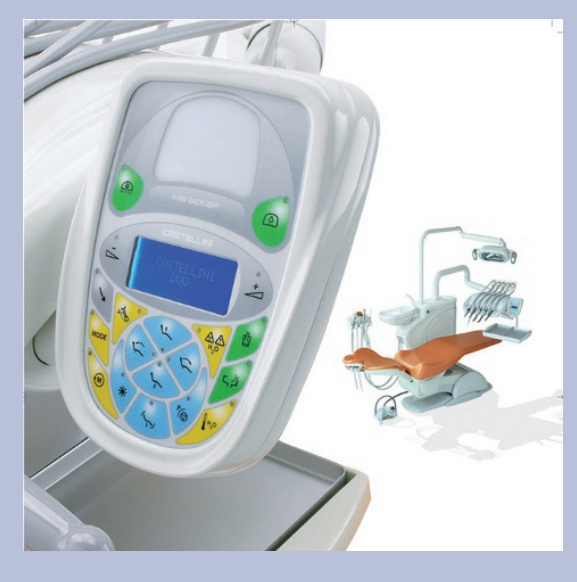

\section{MAKE ORTHODONTIC BONDING EASIER}

To make orthodontic bonding of bands and appliances easier and quicker, a new addition to the range of GC Fuji ORTHO resin-modified glass ionomer cements has been introduced.

GC Fuji Ortho Band is an orthodontic cement made available as a paste in the special Paste Pak cartridge. The unique Paste Pak Dispenser allows for a faster and more accurate dispensing system, which minimises cement waste.
Optimal handling is guaranteed by a film thickness of $3 \mu \mathrm{m}$, a working time of 3 minutes 30 seconds and a setting time of 3 minutes. The final colour of the paste is blue which makes identification of residual cement after de-bonding much easier and quicker.

Further features include no acid etching and problem-free removal without damage to enamel.

Reader response number 59 


\section{DESIGN A STERILISATION ROOM}

Dental Services Direct can work with dentists to design a sterilisation room. They can suggest the correct specification of equipment required and discuss practical considerations such as installing the equipment, as well as giving good practice advice for the use of the room.

In addition, the team is available for planned maintenance to ensure the room is never out of action. The company offers a wide range of equipment

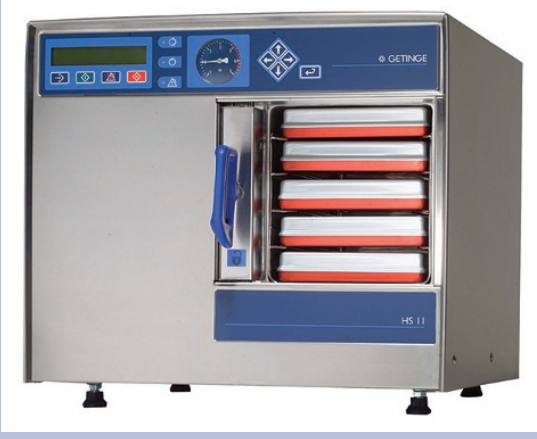

for use in all infection control situations such as the Steelco Dental Washer Disinfectors range. They are an efficient aid for the cleaning and thermal disinfection of all dental instruments and accessories.

You simply take care of loading the instruments and the selection of the cycle, and the rest - pre-wash, washing, thermal disinfection, hot air drying - is taken care of by the machine. At the end of the cycle all the instruments are ready for sterilisation without any further handling.

Another popular range is the Getinge $\mathrm{K}$-series tabletop sterilisers. The standard process (B process) takes 25 minutes and the high-speed process ( $\mathrm{N}$ process) for unwrapped solid instruments takes as little as nine minutes. The rectangular chamber is optimally designed for maximum load capacity so the K-series can sterilise up to ten trays in just 25 minutes.

Reader response number 60

\section{GUIDED SURGERY INSTRUMENTATION}

Biomet $3 \mathrm{i}$ has developed the Navigator System, instrumentation for CT guided surgery. By using the Biomet 3i Navigator System in conjunction with third party CT planning software and surgical guides, clinicians may be able to enhance treatment planning and improve the accuracy of placing Biomet $3 \mathrm{i}$ implants in predetermined locations with control of the hex orientation.

The system provides the instrumentation for CT guided surgery with the Navigator Surgical Kit and Navigator Laboratory Kit. The Navigator Surgical Kit includes easy-to-identify instrumentation that simplifies the process for clinician and staff. Drills are depth-specific with stops built in for definitive preparation. There is no need for adjustable depth control measures, loops, callipers or looking for the right line. Variable prolongation decreases the amount of space needed to perform this type of surgery, which inherently requires longer instrumentation given the added dimension of the surgical guide.
The Navigator Laboratory Kit is used in conjunction with the surgical guide provided by a third party to fabricate provisional restorations prior to the day of surgery.

The kit is comprised of analogue mounts in various lengths that allow laboratory technicians to pour a master cast and orient the analogues as the implants are to be positioned, including hex orientation.

This feature is especially beneficial for single unit cases, cases where abutments are off-angle and cementretained provisionals.

The Navigator System offers compatibility with most existing CT planning software, eliminating the need to purchase dedicated software or to switch from current software. Clinicians have the option to perform a variety of cases including single unit, partial and fully edentulous cases. Surgical flexibility allows clinicians to perform bone, tissue and tooth-supported cases and clinicians may choose from a variety of restorative options.

Reader response number 61
PRECISE AND ACCURATE VIEWING

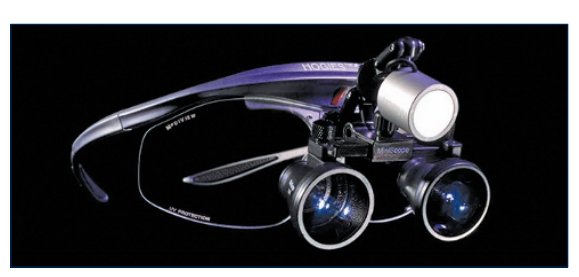

Blackwell Supplies introduces the latest in the Hogies range, MaximEyes Loupes. The loupes are fitted with lenses designed for precise and accurate viewing of the treatment site.

The wearer can achieve the most comfortable posture throughout even the most demanding and time-consuming procedures, with a working distance that rises in increments of $50 \mathrm{~mm}$ from $350 \mathrm{~mm}$ to $500 \mathrm{~mm}$.

The MaximEyes frame design reduces the weight of the loupes on the wearer's nose by $70 \%$, while the multi-adjustment feature lets the wearer adjust the Loupes to suit individual requirements.

Also, the established Hogies MiniScope Loupes are available with $2.5 x$ or $3 x$ magnifications. Features include a lightweight frame that is easily adjustable, and the best patient care is made easier due to the uninterrupted field of vision, working distance of $420 \mathrm{~mm}$ and depth of field.

Reader response number 62

\section{BONDING ORTHO BRACKETS}

BeautyOrtho Bond, known as BOB from Shofu is the first of a new generation of bonding agents developed specifically for bonding orthodontic brackets. Preetching, with the ultimate demineralisation of the tooth structure, is not necessary with BeautyOrtho Bond as the primer is made up of 6-MHP - which chemically adheres to the enamel.

$\mathrm{BOB}$ is a simple and quick to use system providing good handling properties and easy debonding at the end of treatment without damaging the tooth structure. The S-PEG filler component of $\mathrm{BOB}$ releases fluoride on a continuous basis, meaning that the material is constantly re-charged providing ultimate protection for the teeth (as with gics).

Reader response number 63 